\title{
Mitochondrially targeted ceramide LCL-30 inhibits colorectal cancer in mice
}

\author{
F Dahm', A Bielawska², A Nocito', P Georgiev', ZM Szulc², J Bielawski², W Jochum, D Dindo', YA Hannun² \\ and P-A Clavien*,I \\ 'Swiss HPB (Hepato-Pancreato-Biliary) Centre, Department of Visceral and Transplantation Surgery, University Hospital Zurich, Rämistrasse I00, Zurich \\ $\mathrm{CH}-809$ I, Switzerland; ' ${ }^{2}$ Department of Biochemistry and Molecular Biology, Medical University of South Carolina, 173 Ashley Avenue, Charleston, SC \\ 29425, USA; ${ }^{3}$ Department of Pathology, University Hospital Zurich, Rämistrasse 100, Zurich CH-809I, Switzerland
}

\begin{abstract}
The sphingolipid ceramide is intimately involved in the growth, differentiation, senescence, and death of normal and cancerous cells. Mitochondria are increasingly appreciated to play a key role in ceramide-induced cell death. Recent work showed the Cl6-pyridinium ceramide analogue LCL-30 to induce cell death in vitro by mitochondrial targeting. The aim of the current study was to translate these results to an in vivo model. We found that LCL-30 accumulated in mitochondria in the murine colorectal cancer cell line CT-26 and reduced cellular ATP content, leading to dose- and time-dependent cytotoxicity. Although the mitochondrial levels of sphingosine- 1 phosphate (SIP) became elevated, transcription levels of ceramide-metabolising enzymes were not affected. In mice, LCL-30 was rapidly absorbed from the peritoneal cavity and cleared from the circulation within $24 \mathrm{~h}$, but local peritoneal toxicity was doselimiting. In a model of subcutaneous tumour inoculation, LCL-30 significantly reduced the proliferative activity and the growth rate of established tumours. Sphingolipid profiles in tumour tissue also showed increased levels of SIP. In summary, we present the first in vivo application of a long-chain pyridinium ceramide for the treatment of experimental metastatic colorectal cancer, together with its pharmacokinetic parameters. LCL-30 was an efficacious and safe agent. Future studies should identify an improved application route and effective partners for combination treatment.

British Journal of Cancer (2008) 98, 98- 105. doi:10.1038/sj.bjc.6604099 www.bjcancer.com
\end{abstract}

Published online 20 November 2007

(c) 2008 Cancer Research UK

Keywords: colorectal neoplasms; cell death; ceramides; ceramidoids; pharmacokinetics; drug therapy

The last two decades have seen an explosive growth in the understanding of sphingolipid biology. Initially considered inert structural constituents of cell membranes or precursors thereof, sphingolipids have emerged as key messenger and bioactive molecules in a wide range of biological processes (Futerman and Hannun, 2004). The sphingolipid ceramide can be formed by the breakdown of sphingomyelin or through de novo synthesis. It is intimately involved in growth, differentiation, senescence, and death of normal and cancerous cells. Several inductors of cell death, for example, TNF $\alpha$ (Obeid et al, 1993), anthracyclines (Bose et al, 1995), or irradiation (El-Assaad et al, 2003) involve ceramide signalling. Administration of exogenous ceramide also causes cell death in various cancer cell lines (Oh et al, 1998; Bras et al, 2000). It is noteworthy that, many cancer cells have a specific 'sphingolipidphenotype', including lower endogenous ceramide levels (Itoh et al, 2003) and a higher sensitivity to the effects of exogenous ceramide (Selzner et al, 2001). This offers the opportunity to selectively target cancer cells with ceramide compounds.

Mitochondria are increasingly appreciated to play a key role in ceramide-induced cell death. Ceramide treatment of isolated

*Correspondence: Dr P-A Clavien; E-mail: clavien@chir.unizh.ch Revised 22 October 2007; accepted 29 October 2007; published online 20 November 2007 mitochondria leads to the activation of a mitochondrial protein phosphatase (PP2A), which dephosphorylates the antiapoptotic Bcl-2 (Ruvolo et al, 1999) and causes cytochrome $c$ release (Ghafourifar et al, 1999). Furthermore, ceramide has been shown to inhibit mitochondrial complex I (Di Paola et al, 2000) and to induce the formation of reactive oxygen species in mitochondria (Garcia-Ruiz et al, 1997). Targeted delivery of sphingomyelinase to mitochondria, but not to other subcellular compartments, results in bax translocation and the activation of the mitochondrial pathway of apoptosis (Birbes et al, 2001).

The central role of mitochondria in ceramide-induced cell death makes them an alluring target for the specific delivery of ceramide compounds. Naturally occurring ceramides contain a relatively long $\mathrm{N}$-linked fatty acyl chain (14-24 carbon atoms), rendering them practically insoluble in water. Ceramides modified with a $\omega$-pyridinium moiety contain a positive charge delocalised over the $\pi$-electron system (Szulc et al, 2006). These ceramide analogues exhibit a much higher water solubility and preferentially accumulate within the mitochondrial matrix driven by the electrochemical gradient (Novgorodov et al, 2005; Dindo et al, 2006; Senkal et al, 2006). The approach of attacking mitochondria is further supported by the fact that cancer cells' mitochondrial membrane potential tends to be more polarised than that of normal cells (Chen, 1988). 
Despite many pleas for ceramide-based treatment regimens against cancer (Radin, 2003), progression from cell-culture to in vivo applications has been slow, and no clinical trials have been reported to date. Previous work in animal models has shown the ceramidase inhibitor B13 to profoundly suppress the growth of colorectal liver metastases (Selzner et al, 2001) and to reduce the progression of metastatic prostate cancer (Samsel et al, 2004). A short-chain pyridinium ceramide (C6-analogue) was recently shown to inhibit tumour growth in a model of metastatic head and neck squamous cell carcinoma (Senkal et al, 2006). These studies illustrate the large potential of a targeted approach to cancer therapy by interference with ceramide signalling.

Our recent work showed LCL-30, a C16-pyridinium ceramide analogue, to induce cell death in vitro by mitochondrial targeting (Dindo et al, 2006). The aim of the current study was to translate these results to an in vivo model. This study represents the first application of a long-chain pyridinium ceramide in vivo as well as a determination of its tolerability and efficacy in a widely used animal model of metastatic colorectal cancer.

\section{MATERIAL AND METHODS}

\section{Cell culture and biological reagents}

D-erythro-2-N-(16'-(1"'-Pyridinium)-hexadecanoyl)-sphingosine bromide (LCL30) was prepared in the Lipidomics Core of the Medical University of South Carolina (Szulc et al, 2006). CT-26 murine colon carcinoma cells (ATCC, Manassas, VA, USA) were cultured in RPMI Medium (Invitrogen, Basel, Switzerland) supplemented with $10 \%$ fetal bovine serum (PAA Laboratories, Austria), $100 \mathrm{U} \mathrm{ml}^{-1}$ of penicillin, and $100 \mu \mathrm{g} \mathrm{ml}^{-1}$ of streptomycin (Invitrogen). The cells were maintained at $37^{\circ} \mathrm{C}$ in a $5 \% \mathrm{CO}_{2}$ atmosphere. Actinomycin $\mathrm{D}$ and doxorubicin hydrochloride were from Sigma-Aldrich (Buchs, Switzerland). Recombinant human TNF $\alpha$ was purchased from R\&D Systems Inc. (Minneapolis, MN, USA). Caspase- 3 and -8 substrates (Ac-DEVD-AFC and Ac-LETDAFC, respectively) as well as caspase-3- and pan-caspase-inhibitor (Z-DEVD-CHO and Z-VAD-fmk, respectively) were from Alexis Biochemicals (Lausen, Switzerland).

\section{Cell viability assay}

Cells were seeded into 12-well plates at a density of approximately $50 \%$, corresponding to $5 \times 10^{5}$ cells per well and allowed to adhere overnight, before the medium was changed to the specified conditions. The MTT assay was performed as described previously (Dindo et al, 2006). In parallel, cells were detached using $1 \%$ trypsin (Invitrogen) and centrifuged at $800 \mathrm{~g}$. Cell pellets were resuspended in PBS with trypan blue (Sigma-Aldrich) and both stained and unstained cells were counted.

\section{Mitochondrial isolation and determination of cytochrome $c$}

All procedures were performed on ice. Cells were scraped and washed twice in PBS before being resuspended in five volumes of isolation buffer ( $250 \mathrm{~mm}$ sucrose, $20 \mathrm{~mm}$ HEPES, pH 7.5, $10 \mathrm{~mm}$ $\mathrm{KCl}, 1.5 \mathrm{~mm} \mathrm{MgCl}_{2}, 1 \mathrm{~mm}$ EDTA, $1 \mathrm{~mm}$ EGTA, $1 \mathrm{~mm}$ DTT, $0.2 \mathrm{~mm}$ PMSF). Cells were broken by repeated aspiration through a pipette. Centrifugation for $10 \mathrm{~min}$ at $700 \mathrm{~g}$ yielded unbroken cells as well as nuclei. Supernatants were centrifuged for $15 \mathrm{~min}$ at $10000 \mathrm{~g}$ to pellet a crude mitochondrial fraction. Mitochondrial enrichment was confirmed by Western blotting for cytochrome $c$ oxidase complex IV (Abcam, 20E8, Cambridge, UK). Mitochondrial cytochrome $c$ release into the cytosol was assessed quantitatively with the Quantikine enzyme-linked immunosorbent assay kit (R\&D Systems, Abingdon, UK).

\section{ATP measurement}

Cellular ATP content was measured in cellular lysates with the Enliten Luciferase/Luciferin reagent (Promega, Mannheim, Germany) according to the manufacturer's instructions and normalised to protein content.

\section{Caspase-3/-8 activities}

Cells were scraped and lysed $(10 \mathrm{~mm}$ Tris-HCl, $\mathrm{pH} 7.4,2 \mathrm{~mm}$ EDTA, $0.1 \% \mathrm{NP}-40$ ) for $10 \mathrm{~min}$ at $4{ }^{\circ} \mathrm{C}$. After centrifugation for $10 \mathrm{~min}$ at $10000 \mathrm{~g}$, the lysate corresponding to $25 \mu \mathrm{g}$ of protein was incubated for $30 \mathrm{~min}$ at room temperature with or without $1 \mu \mathrm{M}$ caspase-3 inhibitor Z-DEVD-FMK. Then, caspase-3 substrate Ac-DEVD-AFC $(10 \mu \mathrm{M})$ or caspase- 8 substrate Ac-LETD-AFC $(10 \mu \mathrm{M})$ and dithiothreitol (10 mM final concentration) were added, and enzyme activity was monitored by measuring fluorescence at $390_{\mathrm{ex}} / 538_{\mathrm{em}} \mathrm{nm}$ (Biolise software and Fluostar microtiter plate reader, Crailsheim, Germany). Caspase activity was then calculated by determining the relative fluorescence units generated under steady state kinetics from which values of caspase-independent protease activity in the presence of the corresponding inhibitor was subtracted. Actinomycin D and TNF $\alpha$ were used as positive controls.

\section{Animal experiments}

All animal experiments were in accordance with Swiss federal animal regulations and approved by the cantonal veterinary office of Zurich. Specific pathogen-free Balb/c mice 10-12 weeks of age (Harlan, Netherlands), syngeneic with the CT-26 colon carcinoma cell line, were kept on a $12 \mathrm{~h}$ day/night cycle with free access to food and water. Animal health, weight, and food intake were monitored daily, and animals were killed according to predefined criteria (signs of pain, reduction of food intake $>50 \%$, weight loss $>20 \%$ ). For subcutaneous tumour cell inoculations, CT-26 cells, cultured in the exponential growth phase, were treated with trypsin and washed in PBS. Cells were then suspended in serum-free medium, and $200 \mu \mathrm{l}$ (corresponding to $5 \times 10^{5}$ cells) were injected subcutaneously. Therapy commenced after a subcutaneous tumour became detectable, which occurred after $4-5$ days. Tumour growth was monitored daily with a sliding calliper, and tumour volume was calculated according to the ellipsoid formula $4 / 3 \pi \times 1 / 2 \times$ w/ $2 \times w / 2$, where 1 is the length and $w$ is the width. Vehicles for intraperitoneal injection of LCL-30 and doxorubicin were $30 \%$ Cremophore (Sigma-Aldrich) and $\mathrm{NaCl}$ respectively. All animals received an equal number of intraperitoneal injections with active compound or appropriate vehicle controls (daily injections of LCL-30 and weekly injections of doxorubicin). Blood cell counts were determined with a Coulter AcT Diff counter (Beckman Coulter, Nyon, Switzerland). Plasma aspartate aminotransferase (AST), alkaline phosphatase, and creatinine were determined with the serum multiple analyzer (Ektachem DTSCII, Johnson \& Johnson Inc., Rochester, USA).

\section{Histology and immunohistochemistry}

Formalin-fixed tissue was paraffin-embedded, sectioned, and stained with H\&E using standard techniques. For immunohistochemistry, tissue sections were incubated with anti-Ki-67 antibody (NeoMarkers). Pretreatment of sections, antibody incubation, and detection of primary antibody (Ventana DAB iView Kit) were performed on a Nexes immunohistochemistry staining system (Ventana Medical Systems, Tucson, AZ, USA). For CD31 staining, detection of primary antibody was performed with a Histofine staining kit (Nichirei Corporation, Tokyo, Japan) and diaminobenzidine (DAB) as a chromogen. All immunostains were counterstained with hematoxylin. TUNEL staining was performed with the 
in situ cell death detection kit (Roche Applied Science, Rotkreuz, Switzerland) according to the manufacturer's instructions. Ki-67 staining was quantified on 10 images with the analySIS $\wedge \mathrm{D}$ imaging software using a semi-automatic thresholding algorithm (Olympus, Volketswil, Switzerland). Microvascular density was counted on 10 high-power fields of CD31-immunostains.

\section{Enzyme-linked immunosorbent assay (ELISA) for TNF $\alpha$}

TNF- $\alpha$ levels in plasma were determined by ELISA (Quantikine mouse TNF- $\alpha, \mathrm{R} \& \mathrm{D}$ systems, Minneapolis, USA) following the manufacturer's instructions. The lower detection limit of this assay is $5.1 \mathrm{pg} \mathrm{ml}^{-1}$.

\section{Quantitative real-time polymerase chain reaction}

Total RNA was extracted from cells or tissue using TRIzol reagent (Invitrogen) following the manufacturer's instructions. Five micrograms of RNA were reverse transcribed to cDNA using the ThermoScript RT-PCR System (Invitrogen) kit. Quantitative realtime PCR amplification and data analysis were performed using an ABI Prism 7000 Sequence Detector System (PE Applied Biosystems, Rotkreuz, Switzerland). TaqMan gene expression assays (PE Applied Biosystems) were used to quantify mRNA expression of the respective genes. Results were quantified as fold induction in comparison to baseline after normalisation to 18S RNA (TaqMan ribosomal RNA control reagents, PE Applied Biosystems).

\section{Detection of LCL-30 and of endogenous sphingolipids}

Liquid chromatography and mass spectrometry (LC-MS) analysis was performed in the Lipidomics Core of the Medical University of South Carolina on a Thermo Finnigan TSQ 7000 triple quadrupole mass spectrometer operating in a Multiple Reaction Monitoring (MRM) positive ionisation mode. Briefly, cell lysates or tumour homogenate fractions were fortified with internal standards for quantification, and lipids were extracted and analysed as previously described (Bielawski et al, 2006).

\section{Statistical analysis}

Data represent mean \pm s.d. of $n$ independent experiments. MannWhitney $U$-test or one-way ANOVA with Student-NewmanKeuls post hoc testing was used as appropriate to compare groups, using SPSS 12.0 (SPSS Inc., Chicago, USA). A $P$-value below 0.05 was considered to indicate statistical significance.

\section{RESULTS}

\section{LCL-30 elicits cytotoxicity in vitro}

We previously tested the cytotoxicity of LCL-30 on a range of human and murine cancer cell lines (Dindo et al, 2006). For the present study, we focused on the colon cancer cell line CT-26, which can be used as a syngeneic in vivo model of colorectal cancer in Balb/c mice. LCL-30 treatment of CT-26 cells was able to effectively induce cell death in vitro in a dose-dependent manner (Figure 1). A $50 \%$ inhibition of cell viability (IC50) was achieved at $10.6 \mu \mathrm{M}$. Time course experiments with different concentrations showed a time-dependent reduction of cell viability with a steady slope (not shown). These results were confirmed by trypan blue exclusion (not shown).

\section{LCL-30 targets mitochondria}

Ceramide has been detected in mitochondria (Dindo et al, 2006) as well as some ceramide-metabolising enzymes such as ceramide synthase and ceramidase. LCL-30 represents a cationic lipid

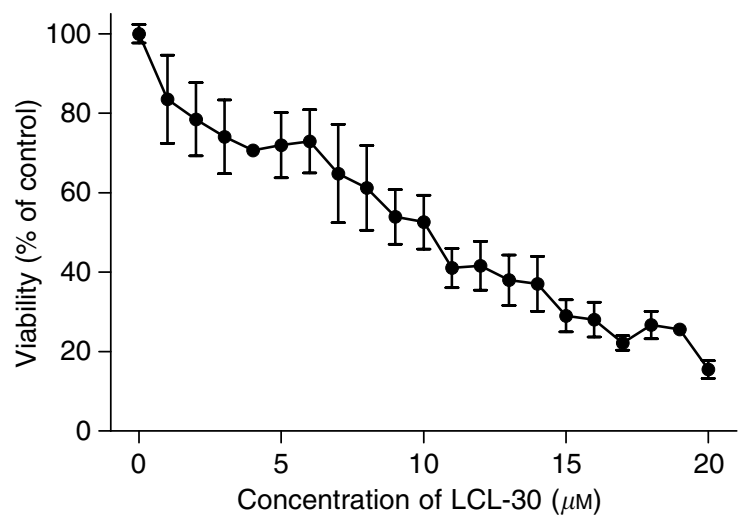

Figure I Cytotoxicity of LCL-30. CT-26 cells were incubated for $24 \mathrm{~h}$ with increasing concentrations of LCL-30, and viability was assessed by the $\mathrm{MTT}$ assay $(\mathrm{IC} 50=10.6 \mu \mathrm{M})$.

designed to be enriched in the positively charged mitochondria. To investigate whether this mitochondrial accumulation occurs in CT-26, cells were treated with the IC50 concentration of LCL-30 $(10 \mu \mathrm{M})$ for up to $8 \mathrm{~h}$. Whole cells and mitochondrially enriched fractions were isolated at different time points and subjected to mass spectrometry, allowing a detailed detection of endogenous sphingolipids as well as LCL-30. As illustrated in Figure 2A, LCL-30 was progressively taken up into cells with levels achieving approximately 0.95 pmole $\mu \mathrm{g}^{-1}$ protein after $4 \mathrm{~h}$ of incubation. Notably, LCL-30 was significantly enriched in the mitochondrial fraction such that its levels in isolated mitochondria reached about 6 pmole $\mu \mathrm{g}^{-1}$ protein by $4 \mathrm{~h}$. Cellular and mitochondrial uptake appeared to level off after $4 \mathrm{~h}$. These results demonstrate significant enrichment of LCL-30 in the mitochondria of CT-26 cells.

\section{LCL-30 induces an endogenous sphingolipid response}

Next, the effects of LCL-30 on endogenous sphingolipids were examined. Cellular levels of total ceramide decreased gradually in response to LCL-30 treatment (Figure 2B), whereas mitochondrial levels peaked after $2 \mathrm{~h}$ before returning to normal. Levels of sphingosine and dihydrosphingosine reacted in an analogous fashion (not shown). Interestingly, cellular and mitochondrial levels of sphingosine-1-phosphate (S1P) rose continuously from almost undetectable levels (Figure 2C), with a more marked rise in the mitochondrially enriched fraction. These experiments demonstrate that LCL-30 enters CT- 26 cells, is enriched in the mitochondrial fraction, and leads to a transient rise of endogenous mitochondrial ceramides as well as a marked rise of mitochondrial S1P.

\section{LCL-30 does not regulate ceramide-metabolising enzymes} at the transcriptional level

The changes in cellular sphingolipid levels in response to treatment, especially the rapid rise of S1P levels, prompted us to analyse the expression levels of key enzymes in the metabolic conversion of ceramide to sphingosine and further to sphingosine1-phosphate. Quantitative real-time PCR was performed for acid ceramidase (Asah1), neutral ceramidase (Asah2), alkaline ceramidase (Asah3), sphingosine kinase 1 (SphK1), and sphingosine kinase 2 (SphK2). Neutral ceramidase was never detectable. None of the expressed enzymes showed any changes in their transcript levels during $8 \mathrm{~h}$ of treatment with LCL-30 (data not shown).

\section{LCL-30 decreases cellular ATP levels}

As the above experiments showed LCL-30 to be enriched in mitochondria, we assessed whether mitochondrial function was 

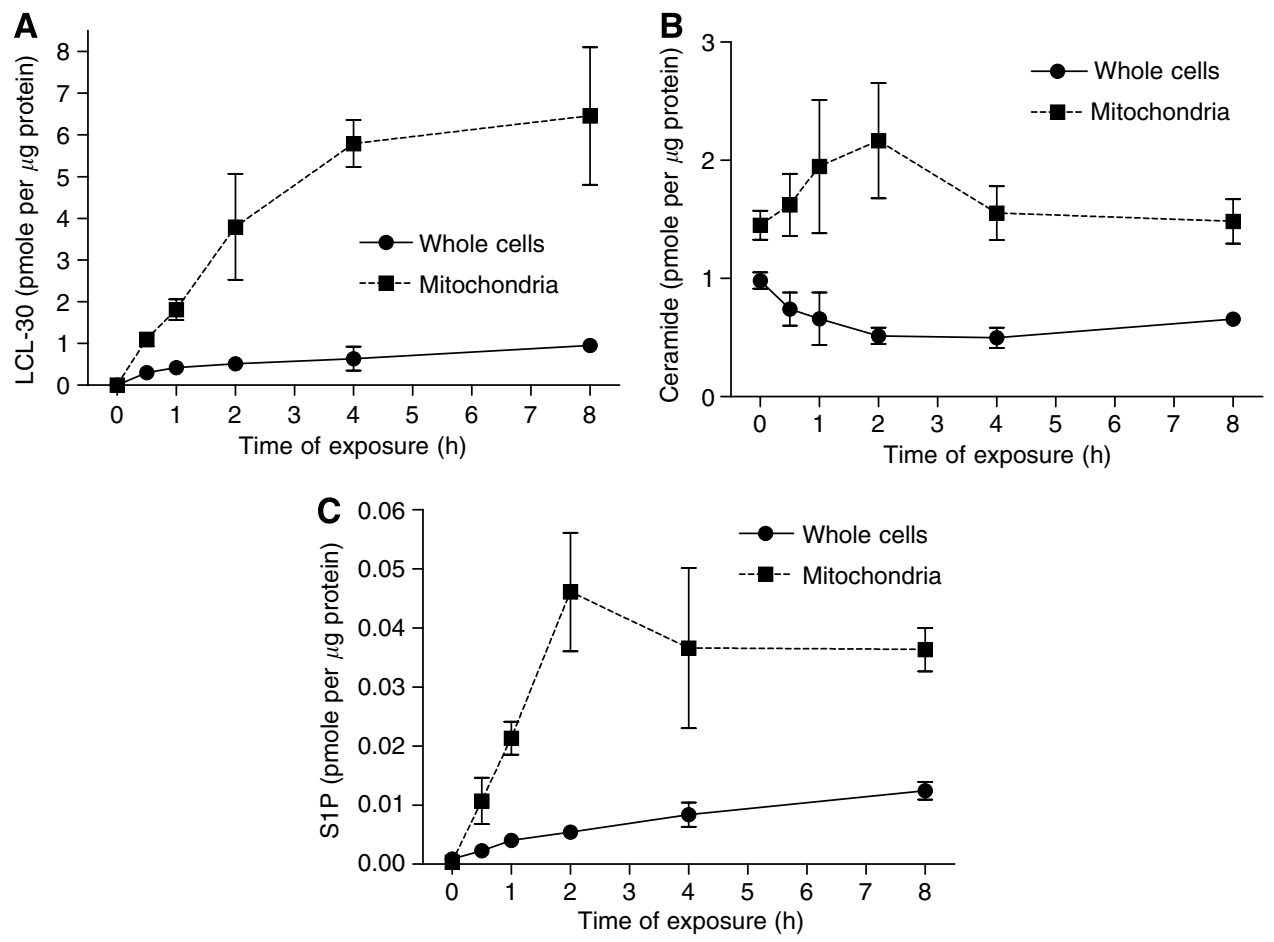

Figure 2 Cellular uptake of LCL-30 (A) and the resulting changes in endogenous ceramide $(\mathbf{B})$ and sphingosine-I-phosphate $(\mathbf{C})$ CT-26 were exposed for the indicated time to $10 \mu \mathrm{M}$ of LCL-30 and then separately analyzed by mass spectrometry for whole cells (squares) and mitochondrially enriched fractions (circles). Results are mean \pm s.d. of $n=3$ and normalised to protein content.
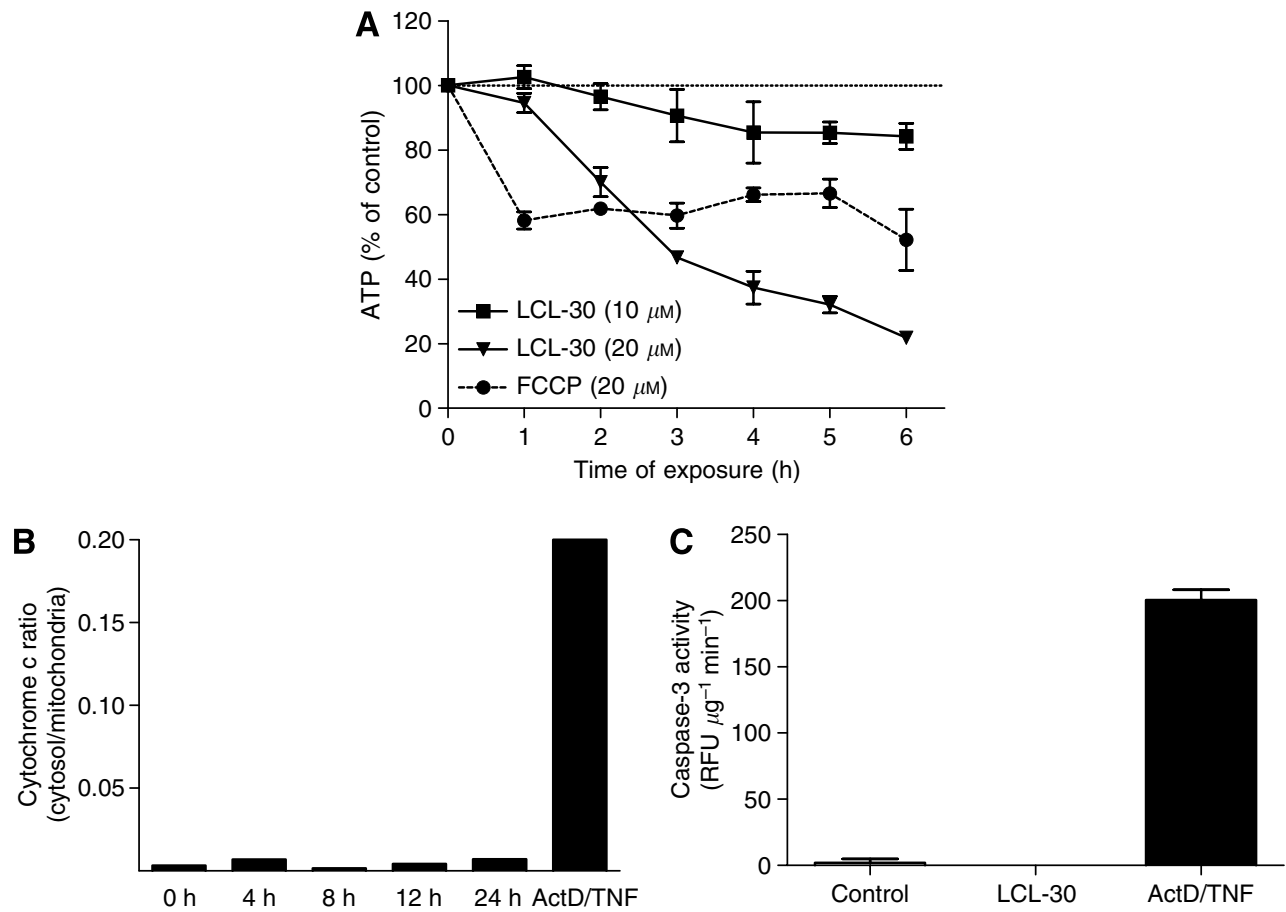

Figure 3 (A) Cellular ATP content during exposure to $20 \mu \mathrm{M}$ FCCP (circles), $10 \mu \mathrm{M}$ LCL-30 (squares) and $20 \mu \mathrm{M} \mathrm{LCL-30} \mathrm{(triangles).} \mathrm{Results} \mathrm{are}$ mean \pm s.d. of $n=4$ and normalised to protein content. (B) Ratio of cytosolic to mitochondrial cytochrome $c$ at different durations of incubation with $10 \mu \mathrm{M}$ LCL-30, as assessed by ELISA. (C) Caspase-3-activity after $6 \mathrm{~h}$ of exposure to $10 \mu \mathrm{M} \mathrm{LCL-30.}$

affected. Cellular ATP levels showed a continuous time- and dosedependent decrease, with different kinetics than the mitochondrial uncoupler FCCP (Figure 3A). Surprisingly, we could not detect cytosolic cytochrome $c$ release by ELISA after 8 or $24 \mathrm{~h}$ of incubation with LCL-30 at a concentration of $10 \mu \mathrm{M}$ (Figure 3B). In line with this observation, we could not detect any caspase 3 (Figure 3C) or caspase 8 activity (data not shown). Actinomycin D and $\mathrm{TNF} \alpha$ (ActD/TNF) were used as positive controls for these assays. Taken together, LCL-30 accumulates in mitochondria of CT-26 cells and decreases ATP production, ultimately causing cell 
death, without detectable cytochrome $c$ release or caspase activation.

\section{Dose-finding and toxicity in mice}

A major aim of this study was to investigate the effects of the long chain pyridinium ceramide LCL-30 in an animal model. To define the toxicity and the tolerable dose, escalating doses of LCL-30 dissolved in $30 \%$ cremophore were administered to mice intraperitoneally. A single dose of $100 \mathrm{mg} \mathrm{kg}^{-1}$ was lethal, while $50 \mathrm{mg} \mathrm{kg}^{-1}$ was lethal after several doses. Toxicity manifested itself by an eosinophilic inflammatory reaction of the peritoneum, followed by fibrinous exudation and fibrous organisation, resulting in severe intraperitoneal adhesions and ileus. No other toxic effects were identified either by histological analysis of internal organs, including brain and bone marrow, or by blood counts and plasma tests (aspartate aminotransferase, alkaline phosphatase,

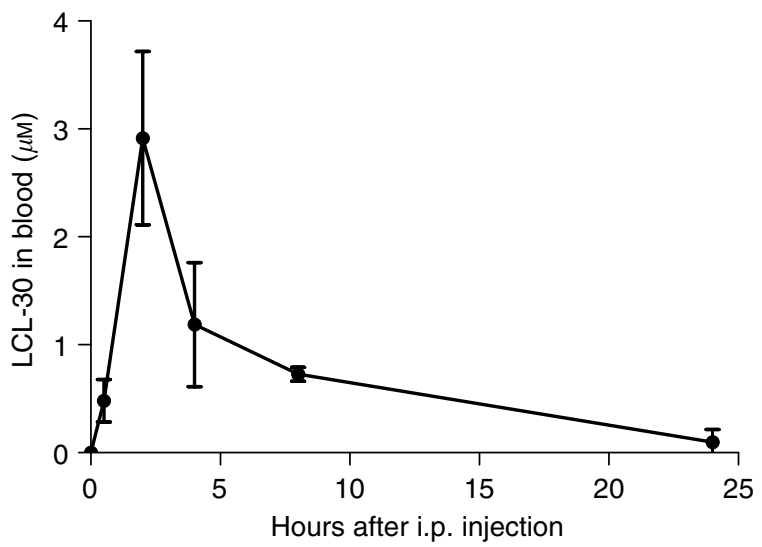

Figure 4 Concentration of LCL-30 in blood after a single intraperitoneal injection of $20 \mathrm{mg} \mathrm{kg}^{-1}$. Results are mean \pm s.d. of $n=4$ and normalised to protein content. and creatinine). Daily injections of $20 \mathrm{mg} \mathrm{kg}^{-1}$ for 1 week were tolerated. This dose was then combined with different doses of doxorubicin. The addition of $6 \mathrm{mg} \mathrm{kg}^{-1}$ doxorubicin to $20 \mathrm{mg} \mathrm{kg}^{-1}$ LCL-30 was also established as safe. Average weight loss in animals undergoing treatment was $6.8 \%$ (control), 9.1\% (doxorubicin), $12.7 \%$ (LCL-30), and $13.9 \%$ (LCL-30 + doxorubicin). None of these animals had to be killed according to the pre-defined criteria. Thus, the in vivo application of LCL-30 caused localised inflammation and did not result in systemic toxicity.

\section{Pharmacokinetics of LCL-30}

After defining the tolerable dose of LCL-30 in vivo, we sought to identify the pharmacokinetic behaviour of this compound. The levels of LCL-30 as well as endogenous sphingolipids were determined by mass spectrometry after a single intraperitoneal dose of $20 \mathrm{mg} \mathrm{kg}^{-1}$ of LCL-30 (Figure 4). LCL-30 was rapidly absorbed from the peritoneal cavity, leading to a peak blood concentration after $2 \mathrm{~h}$. Elimination from blood was almost complete after $24 \mathrm{~h}$. Concentrations were determined separately for plasma and the cellular components of blood: at its peak level, LCL-30 partitioned equally into the aqueous as well as the cellular phase of blood, with slower elimination from the cellular compartment.

\section{Therapeutic efficacy of LCL-30 in subcutaneous tumour grafts}

The next step was the assessment of the therapeutic efficacy of LCL-30 in the treatment of established subcutaneous tumour grafts. Pharmacokinetic data led us to choose a dosing regimen of once per day for LCL-30. After the establishment of solid tumours, animals were randomised to one of four treatment regimens and received an equal number of injections over the course of 1 week. At the beginning of treatment, there were no differences between the animal groups. The growth curves under treatment are shown in Figure 5A; whereas LCL-30 significantly reduced tumour growth, doxorubicin did not cause a significant growth reduction.
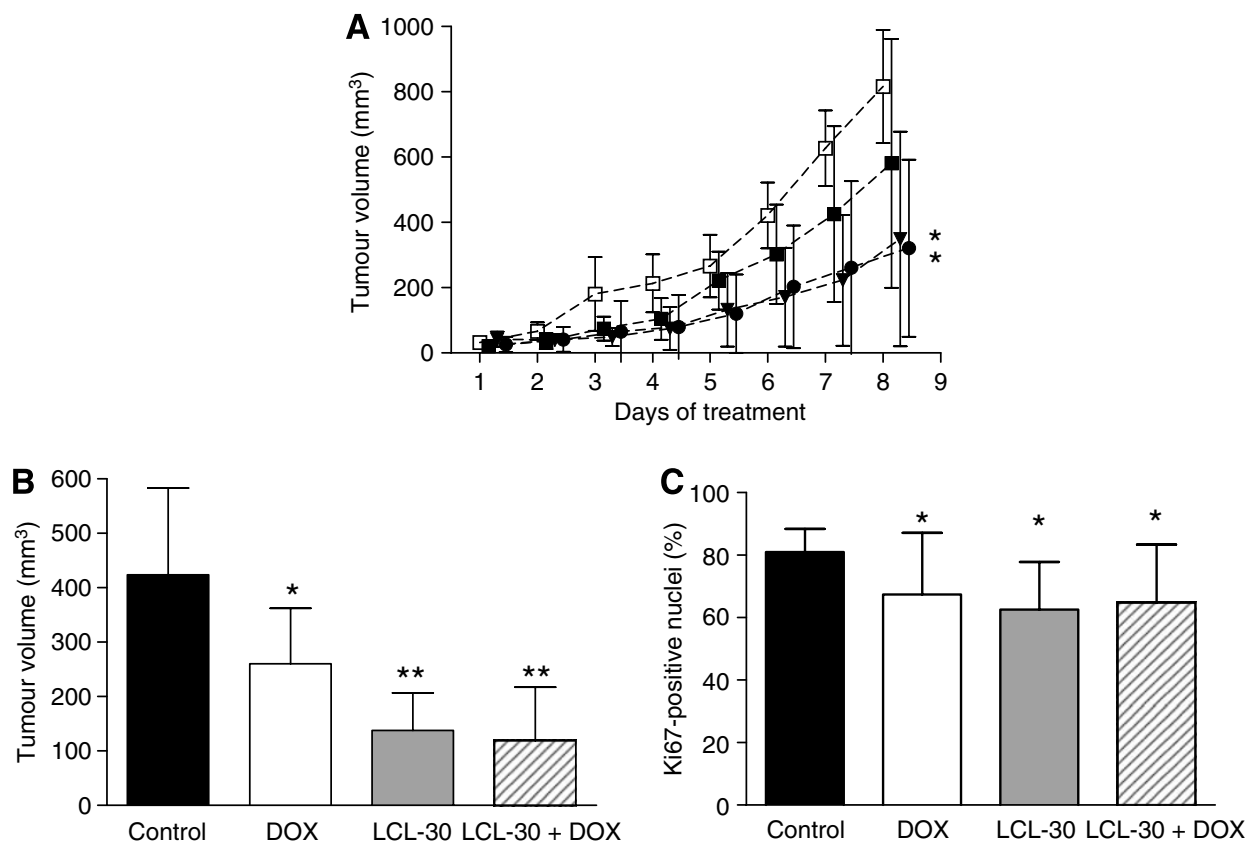

Figure 5 (A) Progression of subcutaneous tumours in animals receiving daily injections with vehicle (open squares), doxorubicin (closed squares), LCL-30 (triangles), or LCL-30 + doxorubicin (circles), *P=0.027 vs control (ANOVA); (B) Volume of explanted tumours after the end of treatment, *P $<0.05$, $* * P<0.01$ vs control (ANOVA with Student-Newman-Keuls post hoc test). (C) Proliferative activity of treated tumours assessed by Ki67 immunohistochemistry, ${ }^{*} P<0.00$ I vs control (ANOVA with Student-Newman-Keuls post hoc test). Results are mean $\pm s . d$. of $n=8$. 
The combination of LCL-30 and doxorubicin did not add to the efficacy of LCL-30 alone. Figure 5B shows the volumes of explanted tumours measured ex-vivo, where doxorubicin-treated tumours were also significantly smaller than controls. Again, LCL-30 was more efficacious than doxorubicin, and the combined treatment did not produce any additive effects. It should be noted that the absolute values of tumour volumes in this protocol are lower due to the systematic overestimation of tumours measured through the skin of live animals. Importantly, the anti-tumour effect could not be explained by an unspecific systemic inflammation, as plasma levels of TNF $\alpha$ remained undetectable in all animals, while positive controls (LPS injection) yielded values of $1098 \pm 261 \mathrm{pg} \mathrm{ml}^{-1}$ (not shown). Therefore, LCL-30 demonstrates significant efficacy against colon cancer in this in vivo model.

\section{Histological assessment of subcutaneous tumours}

Histological assessment of H\&E-stained tumour sections revealed no differences between the groups with respect to tumour morphology or necrotic area. To analyse whether apoptotic cell death plays an important role in tumours, we examined TUNEL stains. There was a low number of single-cell apoptosis in all groups with no differences between groups (not shown). However, the mitotic count was reduced from a baseline of six per high power field (HPF) to five in the doxorubicin group and to four in the LCL-30 and LCL-30 + doxorubicin groups. This led us to use Ki67-immunostaining to further analyse the proliferating tumour cell fraction. The results also showed a reduced proliferative activity of treated tumours (Figure 5C). It is noteworthy that microvascular density (as assessed by CD31-immunostaining) was not different between groups (not shown).

\section{Sphingolipid profiles of subcutaneous tumours}

After completion of treatment, tumour samples were also subjected to mass spectrometry analysis of sphingolipid content. Importantly, $24 \mathrm{~h}$ after the last injection of a 1-week treatment course, LCL-30 was detected at $0.22( \pm 0.09)$ and $0.23( \pm 0.08)$ pmole $\mu \mathrm{g}^{-1}$ protein in the LCL-30 and LCL-30 + doxorubicin-treated groups, respectively. The content of endogenous ceramides decreased in the LCL-30-treated tumours, an effect that was less pronounced after doxorubicin co-treatment (Figure 6A). Similar to the in vitro effects, LCL-30 caused an increase in S1P levels (Figure 6B), while sphingosine levels were lowered by LCL-30 treatment (not shown). Thus, LCL-30 appears to concentrate on tumours even when it has been cleared from the blood with persistent effects on tumour sphingolipids that recapitulate its effects in tissue culture.

\section{DISCUSSION}

This study is the in vivo continuation of our previous experiments with LCL-30, the cationic water-soluble analogue of $\mathrm{C}_{16}$-ceramide, in which we could demonstrate that LCL-30 accumulates in the mitochondria of SW403 colorectal cancer cells and induces mitochondrial swelling, cytochrome $c$ release, caspase activation, and eventually cell death (Dindo et al, 2006). Here, we expand our analysis to the murine colon carcinoma cell line CT-26 cells and use CT-26 cells in syngeneic Balb/c mice as an in vivo model of colorectal cancer.

LCL-30 was cytotoxic for CT-26 cells in a dose- and timedependent fashion, in analogy to other cell lines previously tested (Dindo et al, 2006). Cellular fractionation and mass spectrometric analyses showed LCL-30 to be enriched in the mitochondrial fraction, in line with published data on cationic $\omega$-pyridinium analogues of $\mathrm{C}_{6}$ and $\mathrm{C}_{16}$ ceramide (Novgorodov et al, 2005; Dindo et al, 2006; Senkal et al, 2006). Incubation with LCL-30 led to a doseand time-dependent decrease of cellular ATP-levels, pointing to a
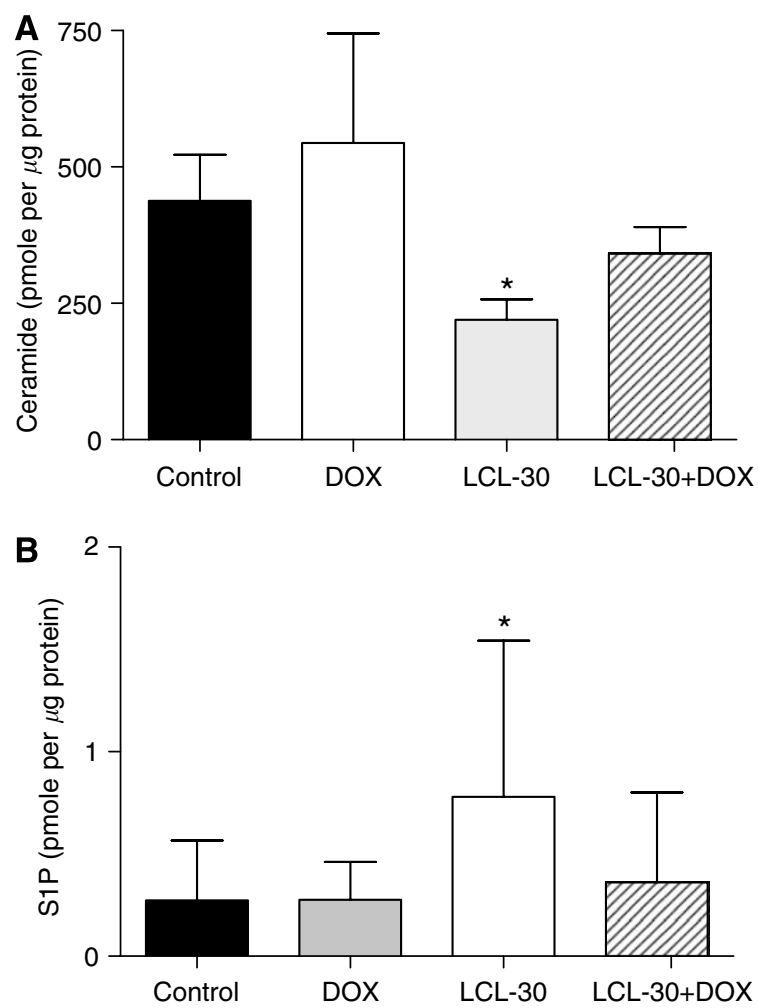

Figure 6 (A) Levels of endogenous ceramide in tumours after I week of treatment with the indicated regimen, $* P=0.098 \mathrm{vs}$ control. (B) Levels of sphingosine-I-phosphate in tumours after I week of treatment with the indicated regimen, ${ }^{*} P=0.045$. Results are mean \pm s.d. of $n=4$ and normalised to protein content.

breakdown of mitochondrial respiration, as already described for the $\omega$-pyridinium $\mathrm{C}_{6}$ analogue LCL-29 (Novgorodov et al, 2005). Yet to our surprise, hallmarks of mitochondrially mediated apoptotic cell death, such as cytochrome $c$ release or caspase activation, could not be detected. The mechanism of LCL-30-mediated cell death in CT-26 remains unclear, although ceramide has been implicated as an endogenous mediator of caspase-independent programmed cell death (Thon et al, 2005). Delineating the differences between cell lines that show caspase activation (SW403) and those without (CT-26) might help define the different mechanisms involved.

Exposure to LCL-30 led to a transient depression of whole-cell ceramide levels, whereas mitochondrial ceramide levels showed a transient rise. This ceramide response is somewhat different from SW403, which show a mitochondrial decrease of ceramide levels (Dindo et al, 2006). Importantly, the rapid and pronounced rise of mitochondrial S1P levels is comparable between both cell lines, raising the possibility of the presence of a mitochondrial sphingosine kinase (SphK). Additional experiments with isomers of LCL-30 have revealed S1P to be derived from endogenous sources and not from the breakdown of LCL-30 (Bielawska A, unpublished). S1P has been primarily regarded as a counter player of ceramide activity, although the intracellular compartmentalisation and the biological context (Ikeda et al, 2003) are important for its biological effects. Future experiments should take intracellular distribution of sphingosine kinase proteins into account. While the activation of SphK1 leads to reduced apoptosis and improved proliferation, activation of SphK2 has been associated with enhanced cell death (Liu et al, 2003), which has been attributed to differential localisation in ER vs cytosol (Wattenberg et al, 2006). Neither SphK1 nor SphK2 has been detected in mitochondria. Nevertheless, there is evidence for additional sphingosine kinase activity (Fukuda et al, 2003), which might be responsible for 
the rise in mitochondrial levels of S1P in response to LCL-30. Alternatively, enhanced levels of S1P might be explained by an inhibition of S1P-degrading enzymes S1P Lyase or S1P phosphatase by LCL-30 (Oskouian et al, 2006). At present, the exact role of S1P produced in response to exogenous treatment with a ceramide analogue remains elusive: it could be an antiapoptotic escape mechanism, a cytotoxic signal, or an epiphenomenon.

Another focus of this study was to assess the safety and efficacy of LCL-30 in an in vivo mouse tumour model. The maximum tolerable dose could be established in dose-escalation studies. Interestingly, dose-limiting toxicity manifested itself as a local peritoneal reaction. The lack of organ-specific toxic effects is encouraging as it carries two important implications. First, it hints at a certain degree of tumour-selectivity of LCL-30, and second, the locally toxic effects of LCL-30 might be circumvented by alternative modes of application.

After a single intraperitoneal injection, LCL-30 reached a peak concentration in blood within $2 \mathrm{~h}$ and was cleared within $24 \mathrm{~h}$. Peak concentrations were lower than LC50 in vitro, although a higher peak between the first two pharmacokinetic sampling points ( $30 \mathrm{~min}$ and $2 \mathrm{~h}$ ) cannot be excluded. Clearance was somewhat slower than for $\mathrm{C}_{6}$ pyridinium, which was already cleared from the circulation after $4 \mathrm{~h}$ by renal excretion (Senkal et al, 2006), suggesting that ceramides with longer acyl chains might be cleared from the circulation more slowly. Such pharmacokinetic behaviour might be beneficial for therapeutic purposes.

Treatment of established subcutaneous tumours over the course of 1 week showed LCL-30 to be an efficacious compound in vivo. Cytotoxic effects on tumours in vivo were less than expected from in vitro experiments, possibly due to insufficient peak concentrations being reached in vivo. Inhibition of tumour proliferation might have been caused by an unspecific inflammatory response to peritoneal injection of a peritoneal irritant. As TNF $\alpha$ could not be detected in the plasma of any animal, this is highly unlikely. Relative ceramide levels were much higher in solid tumours than in cell culture, which might be caused by a different sphingolipid composition of tumour cells growing in vivo. Solid tumours also contain additional cell types, such as stromal or infiltrating blood-derived cells, with a high ceramide content (Dahm et al, 2006). On the basis of in vitro data showing synergistic cytotoxicity of doxorubicin with LCL-30 (Dindo et al, 2006), doxorubicin was also tested alone and in combination treatment. In contrast to the in vitro observation, doxorubicin conveyed no additive effect compared to LCL-30 alone. This might be related to the dosing schedule where LCL-30 was administered daily and doxorubicin, once per week. Weekly administration of doxorubicin was based on established dosing regimens (Yoneda et al, 1999; Dubois et al, 2002).

In summary, we present the first in vivo application of a longchain cationic ceramide for the treatment of experimental metastatic colorectal cancer, together with its pharmacokinetic parameters. Although cytotoxic for CT-26, the mechanism of cell death was different from the previously studied SW403, and doxorubicin did not convey additive effects in vivo. Nevertheless, LCL-30 was an efficacious and safe agent. Future studies should further elaborate on the mechanism of cell death and aim to identify an alternative application route as well as more effective partners for combination treatment.

\section{ACKNOWLEDGEMENTS}

We thank Stefan Heinrich, Gerd Kullak, Daniel Fetz, Peter Gehrig, Riem Ha (University and University Hospital Zurich), Jürgen Schiller, Jan Hengstler (University Leipzig), Besim Ogretmen, Tarek Taha, and Sergei Novgorodov (Medical University South Carolina) for helpful discussions, and Valentin Rousson (Department of Biostatistics, Institute for Social and Preventive Medicine, University of Zurich) for statistical advice. Furthermore, we are grateful to Udo Ungethüm, Marion Bawohl, Claas Börger (University Hospital Zurich), and Barbara Rembiesa (Medical University of South Carolina) for technical assistance. This work was supported by Sassella Foundation, Zurich, Switzerland (to FD) and the National Cancer Institute, Bethesda, MD (Grant IPO1$\mathrm{CA} 097132$ to $\mathrm{AB}$ and $\mathrm{YAH}$ )

\section{REFERENCES}

Bielawski J, Szulc ZM, Hannun YA, Bielawska A (2006) Simultaneous quantitative analysis of bioactive sphingolipids by high-performance liquid chromatography-tandem mass spectrometry. Methods 39: $82-91$

Birbes H, El Bawab S, Hannun YA, Obeid LM (2001) Selective hydrolysis of a mitochondrial pool of sphingomyelin induces apoptosis. FASEB J 15: $2669-2679$

Bose R, Verheij M, Haimovitz-Friedman A, Scotto K, Fuks Z, Kolesnick R (1995) Ceramide synthase mediates daunorubicin-induced apoptosis: an alternative mechanism for generating death signals. Cell 82: $405-414$

Bras A, Albar JP, Leonardo E, de Buitrago GG, Martinez AC (2000) Ceramide-induced cell death is independent of the Fas/Fas ligand pathway and is prevented by Nur77 overexpression in A20 B cells. Cell Death Differ 7: 262-271

Chen LB (1988) Mitochondrial membrane potential in living cells. Annu Rev Cell Biol 4: 155-181

Dahm F, Nocito A, Bielawska A, Lang KS, Georgiev P, Asmis LM, Bielawski J, Madon J, Hannun YA, Clavien PA (2006) Distribution and dynamic changes of sphingolipids in blood in response to platelet activation. J Thromb Haemost 4: 2704-2709

Di Paola M, Cocco T, Lorusso M (2000) Ceramide interaction with the respiratory chain of heart mitochondria. Biochemistry 39: 6660-6668

Dindo D, Dahm F, Szulc Z, Bielawska A, Obeid LM, Hannun YA, Graf R, Clavien P-A (2006) Cationic long-chain ceramide LCL-30 induces cell death by mitochondrial targeting in SW403 cells. Mol Cancer Ther 5: $1520-1529$

Dubois V, Dasnois L, Lebtahi K, Collot F, Heylen N, Havaux N, Fernandez AM, Lobl TJ, Oliyai C, Nieder M, Shochat D, Yarranton GT, Trouet A (2002)
CPI-0004Na, a new extracellularly tumor-activated prodrug of doxorubicin: in vivo toxicity, activity, and tissue distribution confirm tumor cell selectivity. Cancer Res 62: 2327-2331

El-Assaad W, Kozhaya L, Araysi S, Panjarian S, Bitar FF, Baz E, El-Sabban ME, Dbaibo GS (2003) Ceramide and glutathione define two independently regulated pathways of cell death initiated by p53 in Molt- 4 leukaemia cells. Biochem J 376: $725-732$

Fukuda Y, Kihara A, Igarashi Y (2003) Distribution of sphingosine kinase activity in mouse tissues: contribution of SPHK1. Biochem Biophys Res Commun 309: $155-160$

Futerman AH, Hannun YA (2004) The complex life of simple sphingolipids. EMBO Rep 5: $777-782$

Garcia-Ruiz C, Colell A, Mari M, Morales A, Fernandez-Checa JC (1997) Direct effect of ceramide on the mitochondrial electron transport chain leads to generation of reactive oxygen species. Role of mitochondrial glutathione. J Biol Chem 272: 11369-11377

Ghafourifar P, Klein SD, Schucht O, Schenk U, Pruschy M, Rocha S, Richter C (1999) Ceramide induces cytochrome $c$ release from isolated mitochondria. Importance of mitochondrial redox state. J Biol Chem 274: 6080-6084

Ikeda $\mathrm{H}$, Satoh $\mathrm{H}$, Yanase $\mathrm{M}$, Inoue $\mathrm{Y}$, Tomiya T, Arai M, Tejima K, Nagashima K, Maekawa H, Yahagi N, Yatomi Y, Sakurada S, Takuwa Y, Ogata I, Kimura S, Fujiwara K (2003) Antiproliferative property of sphingosine 1-phosphate in rat hepatocytes involves activation of Rho via Edg-5. Gastroenterology 124: 459-469

Itoh M, Kitano T, Watanabe M, Kondo T, Yabu T, Taguchi Y, Iwai K, Tashima M, Uchiyama T, Okazaki T (2003) Possible role of ceramide as an indicator of chemoresistance: decrease of the ceramide content via 
activation of glucosylceramide synthase and sphingomyelin synthase in chemoresistant leukemia. Clin Cancer Res 9: 415-423

Liu H, Toman RE, Goparaju SK, Maceyka M, Nava VE, Sankala H, Payne SG, Bektas M, Ishii I, Chun J, Milstien S, Spiegel S (2003) Sphingosine kinase type 2 is a putative $\mathrm{BH} 3$-only protein that induces apoptosis. J Biol Chem 278: $40330-40336$

Novgorodov SA, Szulc ZM, Luberto C, Jones JA, Bielawski J, Bielawska A, Hannun YA, Obeid LM (2005) Positively charged ceramide is a potent inducer of mitochondrial permeabilization. J Biol Chem 280: 16096-16105

Obeid LM, Linardic CM, Karolak LA, Hannun YA (1993) Programmed cell death induced by ceramide. Science 259: 1769-1771

Oh WJ, Kim WH, Kang KH, Kim TY, Kim MY, Choi KH (1998) Induction of p21 during ceramide-mediated apoptosis in human hepatocarcinoma cells. Cancer Lett 129: 215-222

Oskouian B, Sooriyakumaran P, Borowsky AD, Crans A, Dillard-Telm L, Tam YY, Bandhuvula P, Saba JD (2006) Sphingosine-1-phosphate lyase potentiates apoptosis via p53- and p38-dependent pathways and is downregulated in colon cancer. Proc Natl Acad Sci USA 103: 17384-17389

Radin NS (2003) Designing anticancer drugs via the achilles heel: ceramide, allylic ketones, and mitochondria. Bioorg Med Chem 11: 2123-2142

Ruvolo PP, Deng X, Ito T, Carr BK, May WS (1999) Ceramide induces Bcl2 dephosphorylation via a mechanism involving mitochondrial PP2A. I Biol Chem 274: 20296 - 20300

Samsel L, Zaidel G, Drumgoole HM, Jelovac D, Drachenberg C, Rhee JG, Brodie AM, Bielawska A, Smyth MJ (2004) The ceramide analog, B13, induces apoptosis in prostate cancer cell lines and inhibits tumor growth in prostate cancer xenografts. Prostate 58: 382-393

Selzner M, Bielawska A, Morse MA, Rudiger HA, Sindram D, Hannun YA, Clavien PA (2001) Induction of apoptotic cell death and prevention of tumor growth by ceramide analogues in metastatic human colon cancer. Cancer Res 61: $1233-1240$

Senkal C, Ponnusamy S, Rossi M, Sundararaj K, Szulc Z, Bielawski J, Bielawska A, Meyer M, Cobanoglu B, Koybasi S, Sinha D, Day T, Obeid L, Hannun Y, Ogretmen B (2006) Potent Anti-Tumor Activity of a Novel Cationic Pyridinium-Ceramide Alone or In Combination with Gemcitabine Against Human Head and Neck Squamous Cell Carcinomas In vitro and In vivo. J Pharmacol Exp Ther 317: 1188-1199

Szulc ZM, Bielawski J, Gracz H, Gustilo M, Mayroo N, Hannun YA, Obeid LM, Bielawska A (2006) Tailoring structure-function and targeting properties of ceramides by site-specific cationization. Bioorg Med Chem 14: $7083-7104$

Thon L, Mohlig H, Mathieu S, Lange A, Bulanova E, Winoto-Morbach S, Schutze S, Bulfone-Paus S, Adam D (2005) Ceramide mediates caspaseindependent programmed cell death. FASEB J 19: 1945 - 1956

Wattenberg BW, Pitson SM, Raben DM (2006) The sphingosine and diacylglycerol kinase super family of signaling kinases: localization as a key to signaling function. J Lipid Res 47: 1128-1139

Yoneda J, Killion JJ, Bucana CD, Fidler IJ (1999) Angiogenesis and growth of murine colon carcinoma are dependent on infiltrating leukocytes. Cancer Biother Radiopharm 14: 221-230 